\title{
O DESPERTAR DA AUTOMOTIVAÇÃO NOS ESTUDANTES POR MEIO DE APRENDIZAGEM BASEADA EM PROJETOS COM FOCO NOS OBJETIVOS DE DESENVOLVIMENTO SUSTENTÁVEL
}

DOI: 10.37702/2175-957X.COBENGE.2021.3532

Rafael Amaral Shayani - shayani@unb.br

Universidade de Brasilia

Quadra 2053

71925-000 - Brasilia - DF

Resumo: A adoção de uma educação mais centrada no estudante requer técnicas de motivação, para que seja feita a transição entre a aula expositiva, com o professor como a fonte do conhecimento, e as aulas em que o aluno é o protagonista de seu próprio aprendizado. Empoderar o aluno a promover a melhora do mundo a partir do conhecimento técnico de engenharia é uma forma efetiva de automotivação. A utilização de aprendizagem baseada em projetos, em que os estudantes tenham a liberdade para escolher o seu próprio projeto, desde que relacionados ao Objetivos de Desenvolvimento Sustentável das Nações Unidas, os insere em um esforço mundial de metas nobres, os quais passam a enxergar a importância das disciplinas de engenharia para melhorar o mundo, motivando-os aos estudos. Essa técnica foi aplicada, de forma experimental, em duas turmas da disciplina de conceitos básicos de eletricidade, onde todos os estudantes relataram que essa metodologia os auxiliou a desenvolver diversas competências de engenharia previstas nas Diretrizes Curriculares Nacionais.

Palavras-chave: Aprendizagem baseada em projetos; Educação centrada no aluno; Objetivos de Desenvolvimento Sustentável. 


\section{O DESPERTAR DA AUTOMOTIVAÇÃO NOS ESTUDANTES POR MEIO DE APRENDIZAGEM BASEADA EM PROJETOS COM FOCO NOS OBJETIVOS DE DESENVOLVIMENTO SUSTENTÁVEL}

\section{INTRODUÇÃO}

O presente artigo apresenta os resultados iniciais de uma proposta de abordagem didática que visa estimular o desenvolvimento das competências de engenharia nos alunos, conforme prescrito nas Diretrizes Curriculares Nacionais (DCN) (MEC, 2019), por meio do empoderamento dos estudantes, visando utilizar o conteúdo da disciplina para resolver grandes problemas da humanidade, apresentados pelos Objetivos de Desenvolvimento Sustentável (ODS) das Nações Unidas (ONU, 2015a), por meio da aprendizagem baseada em projetos (PBL). A hipótese trabalhada é que, ao motivar os estudantes a realizar grandes feitos com a aplicação da teoria de engenharia estudada na disciplina, por meio da concepção de um projeto criativo e inovador a ser desenvolvido sob orientação docente, haverá o estímulo à educação mais centrada no aluno, automotivando-os aos estudos.

Ao adotar uma visão holística e humanística do estudante, inspirado pelas palavras de Bahá'u'lláh (1817-1892), pode-se identificar cada aluno como um ser humano essencialmente espiritual, criado nobre por natureza e destinado a levar avante uma civilização em constante evolução. Ao conclamar os estudantes a "cuidar zelosamente das necessidades da era em que vivem e concentrar vossas deliberações em suas exigências e seus requisitos", haverá um estímulo para reconhecer as necessidades dos usuários. Ao aconselhar os alunos a "dedicar os preciosos dias de vossas vidas ao melhoramento do mundo", eles serão chamados a buscar um propósito para os seus estudos, dando mais significado para o conteúdo técnico que será estudado (BAHÁ'U'LLÁH, 2015). O papel crucial do educador é ensiná-los a dedicar suas vidas a assuntos de grande importância e inspirá-los a empreender estudos que beneficiem a humanidade (CUJ, 1981). Tal é o papel do professor: inspirar os alunos para atuarem em benefício da humanidade!

Muitos cursos possuem elevados índices de desistência por parte dos estudantes. Engenharia é um exemplo, pois diversos alunos não compreendem o porquê de possuírem uma carga teórica tão grande de conceitos físicos e matemáticos e, muitas vezes, desistem do curso por ter sua expectativa inicial, de resolver problemas, frustrada por aulas teóricas conceituais complexas e, aparentemente, sem aplicação direta. É importante que os alunos percebam que esse conhecimento os possibilita a atuarem como protagonistas na sociedade, apresentando soluções viáveis, com forte formação técnica, para problemas reais. Quando os professores contextualizam o ensino e empoderam os alunos ao afirmar que, de posse dos conceitos ministrados, eles terão as ferramentas necessárias para propor soluções que ajudam a salvar vidas e melhorar 0 mundo, os estudantes tendem a ficar mais motivado para estudar e passam a nutrir 0 desejo pelo conhecimento (SHAYANI, 2020a).

$E$ importante explorar conceitos fundamentais e aspirações audazes, a fim de desenvolver um engenheiro crítico e reflexivo. Deve-se enfatizar junto aos alunos que eles são potenciais desbravadores das soluções que a sociedade necessita, equipados com o conhecimento que aprendem em cada uma das disciplinas. Este é um tempo para objetivos nobres, elevada determinação e intenso esforço. Os estudantes devem ser chamados a fazer sua contribuição construtiva aos afazeres humanos, dentro de sua área de especialização. As aulas devem possibilitar que os estudantes visualizem a aplicação prática por trás de cada teoria estudada, para que sejam capacitados a propor soluções 
para os problemas que se apresentam diante da sociedade. $\mathrm{O}$ aluno deve vislumbrar $\mathrm{o}$ estudo universitário não como um fim por si só, mas como um meio de melhor se preparar para servir à humanidade, de forma mais justa, humana e sustentável. Essa deve ser a motivação de cada estudante para se dedicar aos estudos com afinco e alegria! Todos os estudantes devem ser convidados a pensar sob essa ótica e se engajar com motivação nas disciplinas, sempre tentando associar o conteúdo ministrado à uma aplicação prática que possa contribuir para a melhora do mundo. Esse deve ser o diálogo principal entre professor e estudante (SHAYANI, 2020b).

Como motivar os estudantes de engenharia a utilizar sua bagagem técnica para resolver grandes problemas da sociedade? A solução reside em tocar profundamente a essência do estudante, empoderando-o e enfatizando sua capacidade e responsabilidade de propor soluções que tenham como foco o ser humano, e não a tecnologia. Para tal, os professores podem contextualizar suas aulas dentro dos Objetivos de Desenvolvimento Sustentável da ONU, onde o aluno será inserido em um arena que exigirá o discernimento necessário para enxergar como o conteúdo técnico estudado poderá ser utilizado para erradicar a pobreza, reduzir desigualdades, gerar energia limpa, combater a mudança do clima, preservar a vida terrestre e aquática, e promover paz e justiça, entre outros objetivos da Agenda 2030. Tal abordagem servirá de motivador para os estudos, e possui aplicações em diversas áreas da engenharia (SHAYANI, 2019).

O relatório sobre engenharia da Organização das Nações Unidas para a Educação, a Ciência e a Cultura, elaborado pela World Federation of Engineering Organizations (WFEO), International Council of Academies of Engineering and Technological Sciences (CAETS) e International Federation of Consulting Engineers (FIDIC), ressalta a necessidade de transformação da educação em engenharia, enfatizando a aplicação, de forma mais efetiva, do conhecimento de engenharia para resolver problemas globais e desafios tais como redução de pobreza, desenvolvimento sustentável e mudanças climáticas. Uma constatação clara desse relatório é que, se os jovens perceberem que a engenharia é parte da solução de grandes problemas globais, ficarão mais interessados e atraídos ao curso (UNESCO, 2010).

A edição 2014 do relatório da UNESCO sobre engenharia alerta que a importante contribuição que a engenharia realiza para a sociedade não está sendo suficientemente enfatizada, o que contribui para as elevadas taxas de evasão, e que a estrutura do curso deve ser modificada para ficar mais interessante para o aluno e aumentar sua motivação, pois é um elemento crítico para o desenvolvimento acadêmico (UNESCO, 2014).

A edição 2021 do relatório da UNESCO leva o título de "Engenharia para o desenvolvimento sustentável" e tem foco no papel da educação em engenharia para avançar os ODSs e lidar com desafios globais. O relatório pontua que a educação em engenharia está mudando do tradicional foco baseado em conhecimento técnico para uma abordagem mais ampla e interdisciplinar de solução de problemas complexos. Enfatiza também a importância da educação centrada no aluno e da aprendizagem baseada em problemas. Questões como paz e justiça devem ser abordadas, o que envolverá mudanças tanto no conteúdo dos cursos quanto no método de ensinoaprendizagem. O relatório relaciona o tipo de problema (óbvio, complicado e complexo) com as competências requeridas, a estrutura do currículo necessária e os métodos de ensino-aprendizagem a serem utilizados. Problemas complexos, tais como os desafios de sustentabilidade, requerem interdisciplinaridade e reorganização do currículo. Estudos indicam que a adoção de PBL aumenta o grau de consciência sobre sustentabilidade entre os estudantes de engenharia, além de contribuir para aumentar a motivação para o aprendizado, reduzindo a evasão e melhor desenvolvendo as competências. Como recomendação, o relatório enfatiza a importância de investimentos em pesquisa 
relacionada à educação em engenharia visando promover e estimular o desenvolvimento de técnicas pedagógicas e de ensino e aprendizagem de forma sistematizada, com foco na interdisciplinaridade e resolução de problemas complexos utilizando educação centrada no aluno e PBL (UNESCO, 2021).

A importância da adoção de novos paradigmas multidisciplinares e interdisciplinares na educação em engenharia foi abordada por Shayani (2020), o qual enfatiza que a educação tradicional, com foco em tecnologias, deve dar lugar à uma educação com foco no ser humano, com visão mundial dos problemas, baseada em justiça social, considerando os custos ambientais e sociais, e oferecendo o conhecimento que o aluno aprende como uma ferramenta para promover o desenvolvimento da humanidade (SHAYANI, 2020c).

A metodologia aqui proposta tem como objetivo motivar os estudantes por meio de projetos de engenharia que visam objetivos nobres e que tenham potencial de grande impacto positivo social e ambiental, com foco nos Objetivos de Desenvolvimento Sustentável. Ela foi aplicada, em caráter de teste, à disciplina de conteúdo básico de eletricidade, ofertada aos estudantes das engenharias Ambiental, Civil, Mecânica e Química da Universidade de Brasília. As próximas seções apresentam a contextualização dada à disciplina, a estrutura do método aplicado, o resultado obtido com os estudantes e a avaliação de como as características de engenharia foram trabalhadas nos estudantes.

\section{CONTEXTO DA DISCIPLINA DE ELETRICIDADE BÁSICA}

A disciplina "Eletricidade Básica" aborda conteúdo teórico de geração de energia (lei de Faraday), transmissão de energia (funcionamento de transformadores e perdas por efeito Joule), distribuição (sistemas monofásicos e trifásicos, com as respectivas ligações delta e estrela) e consumo (funcionamento de motores elétricos, modelagem de resistor, indutor e capacitor e correção de fator de potência). Trata-se de disciplina teórica com elevada carga técnica de modelagem de sistemas elétricos por meio de equações diferenciais e de matemática envolvendo números imaginários.

A contextualização apresentada aos estudantes é de que a eletricidade é uma forma de transmissão de energia, que será convertida em outras formas energéticas (mecânica ou térmica, por exemplo) para acionar equipamentos de engenharia. Dessa forma, os conceitos teóricos podem ser aplicados a qualquer projeto de engenharia que utilize eletricidade, permitindo, assim, uma vasta gama de aplicações em diversas áreas.

Há uma necessidade urgente de formação de engenheiros com visão holística e humanista, críticos, reflexivos, criativos, que sejam capazes de reconhecer as necessidades dos usuários, formular, analisar e resolver, de forma criativa, os problemas de Engenharia, adotando perspectivas multidisciplinares e transdisciplinares em sua prática, considerando os aspectos globais, políticos, econômicos, sociais, ambientais e culturais, atuando com isenção e comprometimento com a responsabilidade social e com o desenvolvimento sustentável. Tais características, prescritas nas DCNs, são especialmente necessárias na área energética, foco da disciplina de eletricidade básica.

A matriz energética mundial está, a cada ano, aumentando a emissão de gases de efeito estufa e agravando a crise climática (ONU, 2020). Apesar de conferências ambientais serem realizadas com grande intensidade, desde 1992, nota-se que, nos últimos 30 anos, o setor energético continua predominantemente com o mesmo perfil, baseado no crescimento de fontes fósseis, com crescimento ainda tímido de fontes renováveis de energia. Tal característica está relacionada, entre outros aspectos, com a formação de engenharia, a qual, muitas vezes, está focada em transmitir aos estudantes como as fontes energéticas funcionam, e quais são as tradicionalmente mais utilizadas, 
tais como petróleo e gás natural, do que desenvolver o senso crítico dos problemas climáticos associados, ou o senso criativo para propor soluções diferentes do usualmente adotado.

O sistema energético deve ser reestruturado a fim de permitir o crescimento energético dos países em desenvolvimento e, ao mesmo tempo, reduzir as emissões de gases de efeito estufa que agravam a crise climática. Diversos organismos internacionais alertam que a estabilização da concentração de gases de efeito estufa em baixos níveis requer uma transformação fundamental do sistema de geração de energia (IPCC, 2014); deve haver colaboração internacional para remodelar o sistema energético mundial (ONU, 2019a); e o atingimento da meta de emissões líquidas nulas de gases de efeito estufa requer uma transformação radical na forma como a energia é gerada, transformada e utilizada (IEA, 2020).

O estudo das mudanças climáticas, em todos os cursos, é uma oportunidade para que os alunos possam se engajar em um fabuloso desafio da atualidade e exercitar as habilidades de engenharia requeridas pela DCN. As mudanças climáticas são um complexo processo global, que necessita de visão holística e humanista, crítica e reflexiva, e que depende de soluções que considerem dimensões ambientais, sociais, políticas e econômicas, de forma multidisciplinar e interdisciplinar. Trata-se de um propício "estudo de caso" que pode ser abordado em todos os cursos, e que os estudantes precisarão expandir sua visão para propor, dentro de sua área de atuação, soluções práticas para esse grande desafio (SHAYANI, 2021).

A Figura 1 ilustra o crescimento das emissões de gases de efeito estufa pelo setor energético, em especial por conta de geração de eletricidade e calor, processos industriais e transporte, que são temas inerentemente relacionados à engenharia. Essa contextualização expõe a necessidade de novas soluções de engenharia, e convida os estudantes a refletirem sobre tal assunto.

Figura 1 - Crescimento das emissões de CO2 pelo setor energético mundial por setor, indicando sua relação direta com temas de engenharia (IEA, 2021 - modificado).

\section{$\mathrm{CO}_{2}$ emissions by sector, World 1990-2018}

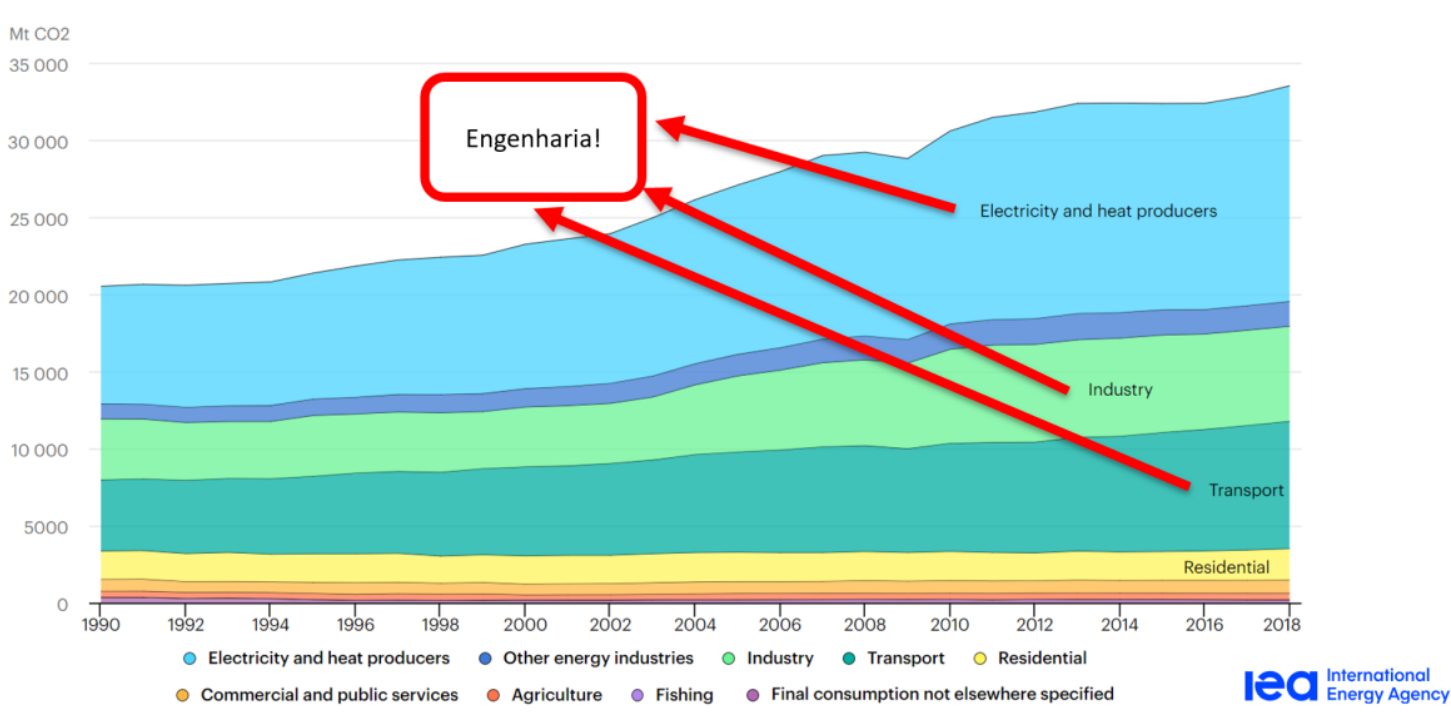


Outro aspecto diretamente relacionado com aplicações de engenharia que envolvem sistemas energéticos é relacionado ao bem-estar da população. As estatísticas apresentadas a seguir apresentam um panorama com diversas oportunidades de projetos de engenharia, provocando uma inquietação ao ser dialogada com os estudantes, motivando-os a utilizar o conhecimento técnico do curso para modificar essa realidade, com soluções criativas e inovadoras (ONU, 2019b):

- 736 milhões de pessoas vivem em extrema pobreza;

- 821 milhões de pessoas estão desnutridas;

- Mais da metade das escolas na África subsariana não tem acesso à água potável ou local para lavar as mãos;

- 785 milhões de pessoas não tem serviço de água potável;

- 673 milhões de pessoas não tem serviço de esgoto;

- 840 milhões de pessoas não tem acesso à eletricidade;

- bilhões de pessoas não tem coleta de lixo;

- 90\% dos moradores urbanos respiram ar poluído;

- A temperatura média global já subiu 1 grau; e

- A acidez dos oceanos já aumentou $26 \%$.

Tal contextualização permite que diversos projetos criativos e inovadores sejam concebidos pelos alunos, dentro do tema eletricidade.

\section{MÉTODO DE ENSINO-APRENDIZAGEM PROPOSTO}

A metodologia proposta consiste em utilizar aprendizagem baseada em projetos para motivar os estudantes a desenvolverem as competências de engenharia. A característica distintiva desta metodologia de ensino-aprendizagem é desafiar 0 estudante, desde o primeiro dia de aula, a conceber um projeto que vise resolver um problema de grande impacto global, social e ambiental, utilizando o conhecimento técnico da disciplina. A forma de escolha do projeto a ser desenvolvido é também um diferencial da metodologia: o projeto deve ser definido pelo próprio grupo de estudantes, e deve ter relação com o seu curso de formação (engenharia Ambiental, Civil, Mecânica ou Química) e, necessariamente, utilizar eletricidade, para ter aplicação direta com a disciplina. $O$ fato dos próprios estudantes escolherem o tema que mais lhes agrada é importante para a motivação.

Visando estimular projetos que necessitem de perspectivas multidisciplinares e transdisciplinares em sua prática, considerando aspectos globais, políticos, econômicos, sociais, ambientais e culturais, os alunos devem ficar cientes dessas características. $O$ seguinte conteúdo é apresentado aos estudantes nas aulas iniciais:

- As diretrizes curriculares nacionais (DCN), para que eles fiquem cientes das características que devem ser desenvolvidas por eles;

- O juramento da engenharia, a ser recitado no dia da colação de grau;

- Os Objetivos de Desenvolvimento Sustentável (ODS) das Nações Unidas, apresentado aos estudantes como as necessidades dos usuários, que devem ser formulados, analisados e resolvidos, de forma criativa, pela Engenharia; e 
- Os novos paradigmas apresentados pelo Acordo de Paris (ONU, 2015b), os quais falam da importância de desenvolver capacidades para utilizar abordagens não mercadológicas integradas, holísticas e equilibradas, no contexto do desenvolvimento sustentável e da erradicação da pobreza, considerando direitos humanos, o direito à saúde, os direitos dos povos indígenas, comunidades locais, migrantes, crianças, pessoas com deficiência e pessoas em situação de vulnerabilidade, o direito ao desenvolvimento, bem como a igualdade de gênero, empoderamento das mulheres e a igualdade intergeracional.

Após a apresentação desse arcabouço inicial, todos os alunos são solicitados a fazer uma redação, visando estimular a comunicação eficaz na forma escrita, sobre o papel da engenharia para resolver os grandes desafios da humanidade, citando exemplos relacionados ao seu curso e que envolvam eletricidade. Ao serem solicitados a refletir sobre o tema, a motivação para empenhar-se no estudo da engenharia é suscitada em suas mentes, pois percebem o quanto poderão contribuir para a sociedade ao dispor de uma forte formação técnica.

Ao levantar temas nobres e importantes dos Objetivos de Desenvolvimento Sustentável, tais como erradicação da pobreza, fome zero, água limpa e saneamento, energia acessível e limpa, redução das desigualdades, cidades e comunidades sustentáveis, consumo e produção responsáveis, combate às alterações climáticas, entre outros, os alunos passam a ficar inspirados e automotivados para a disciplina, pois um convite para mudar o mundo afigura-se muito mais atrativo do que uma matrícula em uma disciplina obrigatória com muitas equações e abstrações elétricas e magnéticas.

Após escolher o tema do projeto, cada grupo deve promover sua contextualização utilizando dados reais disponíveis na internet. Essa abordagem de projeto não estruturado visa desenvolver diversas habilidades de engenharia pois, ao invés de receberem um enunciado preparado pelo professor, com todas as informações necessárias para resolvêlo, os estudantes precisarão conhecer a realidade dos usuários para apresentar uma proposta de projeto de engenharia que seja tecnicamente viável, estimulando-os a formular os problemas de engenharia. E necessário escolher um local real de aplicação e definir o público que será beneficiado pela solução a ser proposta.

Tal trabalho dos discentes ocorre sob a efetiva orientação docente. Visando fornecer uma avaliação formativa, cada grupo apresenta, aproximadamente no meio do curso, um esboço de sua solução, com a contextualização e os cálculos de potência e energia requeridos pela aplicação. Para tanto, são necessários apenas conhecimentos básicos de física, tais como equações de energia cinética e potencial, além de elevada criatividade e inovação. O professor revisa o esboço e apresenta considerações, tanto de ordem matemática quanto de aspectos multidisciplinares e transdisciplinares, para que os estudantes possam aprimorar o projeto.

Ao final da disciplina, todos os grupos mostram seu projeto, tanto na forma escrita, contendo o memorial de cálculo, quanto na forma oral, o qual é apresentado para todos os colegas da turma. No memorial de cálculo deve constar a aplicação de todas as equações estudadas na disciplina.

Durante a apresentação dos grupos, os demais colegas também participam de forma ativa. É informado aos estudantes que uma forma de avaliar se está efetivamente absorvendo o conteúdo da disciplina é conseguir entender tecnicamente as soluções propostas pelos colegas. Assim, o grupo que está apresentando assume o papel de engenheiros mostrando a solução para possíveis investidores, e os colegas de turma assumem o papel de engenheiros que estão avaliando a solução para recomendá-la, ou não, ao possível investidor. Ao final de cada apresentação, todos os alunos preenchem 
um curto questionário, onde informam se entenderam a proposta e os cálculos elétricos, e com um campo dissertativo para pontuar suas impressões a respeito do projeto. Ao término da apresentação, os comentários dos colegas são consolidados pelo professor e compartilhados com a equipe que apresentou, como forma de retroalimentação sobre a impressão que seu projeto causou aos colegas.

No decorrer das aulas expositivas, diversos aspectos relacionados ao papel da engenharia são sistematicamente apresentados ao aluno, intercalados com o conteúdo técnico, para nutrir, durante todo o curso, a importância de desenvolverem soluções criativas e inovadoras.

\section{$4 \quad$ RESULTADOS}

A metodologia proposta foi aplicada a duas turmas de Eletricidade Básica dos cursos de Engenharia da Universidade de Brasília, no segundo semestre de 2019 (20 estudantes) e no primeiro semestre de 2020 (21 estudantes). Trata-se de disciplina obrigatória de 60 horas, ministrada para estudantes de Engenharia Ambiental (6o período), Engenharia Civil (8o período), Engenharia Mecânica (6o período) e Engenharia Química (5o período). Os pré-requisitos são Cálculo 3 e Introdução à Álgebra Linear.

A aplicação da metodologia fez com que a disciplina tivesse, aproximadamente, 10 horas reservadas para motivação aos estudantes, 40 horas de conteúdo expositivo e 10 horas dedicadas à apresentação dos projetos. Apesar do tempo de exposição de conteúdos técnicos ter sido reduzido em 33\%, a motivação em conceber o projeto estimulou a educação mais centrada no aluno, o qual teve a oportunidade de entender o conteúdo de forma ativa durante o projeto.

Os projetos foram definidos pelos estudantes e o relatório final abordou os principais conceitos teóricos aplicados à disciplina, contemplando: a demanda da sociedade que foi trabalhada, a solução proposta e como foram feitos os cálculos de potência e energia, a modelagem elétrica da aplicação (como uma impedância), justificando a escolha dos componentes elétricos, as potências ativa e reativa, informação de como deverá ser a rede elétrica de corrente alternada: monofásica ou trifásica, delta ou estrela, baixa tensão ou alta tensão; se transformadores deverão ser utilizados, como será a correção do fator de potência, e a inclusão de dispositivos de acionamento e proteção conectados ao aterramento, além de, necessariamente, calcular as correntes elétricas que circularão pelos condutores. A apresentação do projeto aborda o mesmo conteúdo de uma prova porém, nesse caso, ao invés do professor fornecer o enunciado pronto, cada grupo elabora seu próprio enunciado por meio do projeto, escolhendo aplicações que os motivam a resolver. A fixação do conteúdo ocorre de forma mais sólida nessa situação.

Os seguintes projetos teóricos foram desenvolvidos pelos estudantes, com aplicações práticas envolvendo os conceitos básicos de eletricidade:

- Tratamento de água em regiões afetadas por desastres naturais;

- Utilização da água da chuva para combater incêndios;

- Bombas submersas a fim de evitar alagamentos;

- Mini hidrelétrica para abastecimento de água e eletricidade para escola;

- Utilização de placas fotovoltaicas para o funcionamento de bombas em estações de tratamento de esgoto;

- Utilização de um sistema de molas e placas no piso para conversão de energia potencial gravitacional em energia elétrica;

- Tratamento de águas cinzas a nível doméstico; 
- Sistema de reaproveitamento de agua para descarga de vaso sanitário com alimentação feita por paineis fotovoltaicos;

- Tratamento de esgoto utilizando biodigestor, com aproveitamento de biogás para geração de energia elétrica;

- Sistema de aquaponia;

- Abastecimento de água e aproveitamento de águas pluviais;

- Reciclagem de resíduos gerados na construção civil;

- Drenagem de fontes de água para combate de incêndios;

- Quadra de esportes eletricamente autossustentável;

- Produção de energia pela queima de resíduos sólidos;

- Unidade simplificada de tratamento de água; e

- Abastecimento de água e energia para comunidade rural.

A diversidade de projetos apresentados permitiu que os estudantes vissem a aplicação do conteúdo de eletricidade estudado no curso de diversas formas, galvanizando seu entendimento.

Após o término de todas as apresentações, os estudantes foram solicitados a preencher um questionário avaliando como a disciplina contribuiu para o desenvolvimento das competências de engenharia. As perguntas estão apresentadas no Quadro 1. O resultado é apresentado na Tabela 1.

A análise dos resultados indica que $98,7 \%$ das respostas aprovam a metodologia utilizada, indicado que trabalha, de forma consciente, as competências de engenharia nos estudantes. As competências mais utilizadas, na elaboração do projeto, foram: reconhecer as necessidades dos usuários, formular, analisar e resolver, de forma criativa, os problemas de Engenharia; e atuar com isenção e comprometimento com a responsabilidade social e com o desenvolvimento sustentável.

Quadro 1 - Perguntas do questionário.

\begin{tabular}{|c|l|}
\hline No. & Pergunta \\
\hline 1 & $\begin{array}{l}\text { Aproveitei ao máximo a oportunidade proporcionada pela elaboração do projeto para desenvolver } \\
\text { visão holística e humanista, ser crítico, reflexivo, criativo, cooperativo e ético e com forte formação } \\
\text { técnica. }\end{array}$ \\
\hline 2 & $\begin{array}{l}\text { Aproveitei ao máximo a oportunidade proporcionada pela elaboração do projeto para desenvolver } \\
\text { a aptidão de pesquisar, desenvolver, adaptar e utilizar novas tecnologias, com atuação inovadora } \\
\text { e empreendedora. }\end{array}$ \\
\hline 3 & $\begin{array}{l}\text { Aproveitei ao máximo a oportunidade proporcionada pela elaboração do projeto para reconhecer } \\
\text { as necessidades dos usuários, formular, analisar e resolver, de forma criativa, os problemas de } \\
\text { Engenharia. }\end{array}$ \\
\hline 5 & $\begin{array}{l}\text { Aproveitei ao máximo a oportunidade proporcionada pela elaboração do projeto para adotar } \\
\text { perspectivas multidisciplinares e transdisciplinares em sua prática. }\end{array}$ \\
\hline 5 & $\begin{array}{l}\text { Aproveitei ao máximo a oportunidade proporcionada pela elaboração do projeto para considerar } \\
\text { os aspectos globais, políticos, econômicos, sociais, ambientais, culturais e de segurança e saúde } \\
\text { no trabalho. }\end{array}$ \\
\hline 6 & $\begin{array}{l}\text { Aproveitei ao máximo a oportunidade proporcionada pela elaboração do projeto para atuar com } \\
\text { isenção e comprometimento com a responsabilidade social e com o desenvolvimento sustentável. }\end{array}$ \\
\hline
\end{tabular}


Tabela 1 - Resposta dos estudantes às perguntas apresentadas no Quadro $1(n=41)$.

\begin{tabular}{|l|c|c|c|c|c|}
\hline & $\begin{array}{c}\text { Concordo } \\
\text { totalmente [\%] }\end{array}$ & $\begin{array}{c}\text { Concordo } \\
\text { parcialmente [\%] }\end{array}$ & $\begin{array}{c}\text { Nem concordo } \\
\text { nem discordo [\%] }\end{array}$ & $\begin{array}{c}\text { Discordo } \\
\text { parcialmente [\%] }\end{array}$ & $\begin{array}{c}\text { Discordo } \\
\text { totalmente [\%] }\end{array}$ \\
\hline Resposta 1 & 71 & 29 & 0 & 0 & 0 \\
\hline Resposta 2 & 71 & 29 & 0 & 0 & 0 \\
\hline Resposta 3 & 83 & 17 & 0 & 0 & 0 \\
\hline Resposta 4 & 76 & 22 & 2 & 0 & 0 \\
\hline Resposta 5 & 59 & 39 & 2 & 0 & 0 \\
\hline Resposta 6 & 83 & 15 & 2 & 0 & 0 \\
\hline
\end{tabular}

\section{CONCLUSÃO}

A mudança da tradicional aula expositiva, em que o professor é a fonte do conhecimento, para uma educação mais centrada no aluno, requer a adoção de estratégias de motivação, para que o estudante seja estimulado a buscar as informações necessárias para resolver problemas complexos, não estruturados, a fim de melhor desenvolver as competências requeridas na engenharia. A adoção de projetos de engenharia com objetivos nobres, empoderando os alunos a promoverem a melhora do mundo ao serem inseridos em um esforço global para atingir os Objetivos de Desenvolvimento Sustentável, mostrou-se uma técnica promissora, pois todos os estudantes que passaram por essa experiência relataram que os auxiliou no desenvolvimento das competências. O resultado é especialmente relevante por ser uma disciplina de eletricidade voltada para estudantes dos cursos de engenharias Ambiental, Civil, Mecânica e Química, os quais foram motivados a relacionar a matéria ao seu próprio curso, ao terem a liberdade de escolher o projeto que gostariam de desenvolver. Tal técnica é um esforço no sentido de indicar como as disciplinas podem ter foco em competências e, ao mesmo tempo, estimular os alunos a estudarem o conteúdo técnico associado, alinhado às Diretrizes Curriculares Nacionais.

\section{REFERÊNCIAS}

BAHÁ'U'LLÁH. Seleção dos escritos de Bahá'u'lláh. Mogi Mirim: Editora Bahá'í, 2015.

CASA UNIVERSAL DE JUSTIÇA (CUJ). Educação Bahá'í - Uma compilação. Mogi Mirim: Editora Bahá'í, 1981.

INTERGOVERNMENTAL PANEL ON CLIMATE CHANGE (IPCC). Mitigation of climate change. 2014. Disponível em: https://www.ipcc.ch/report/ar5/wg3/. Acesso em: 06 mai. 2021.

INTERNATIONAL ENERGY AGENCY (IEA). Energy Technology Perspectives. 2020. Disponível em: https://www.iea.org/reports/energy-technology-perspectives-2020. Acesso em: 06 mai. 2021.

Data and statistics. 2021. Disponível em: https://www.iea.org/data-and-statistics. Acesso em: 06 mai. 2021.

MINISTÉRIO DA EDUCAÇÃO (MEC). Conselho Nacional de Educação. Câmara de Educação Superior. Resolução No. 2, de 24 de abril de 2019. 
ORGANIZAÇÃO DAS NAÇÕES UNIDAS (ONU). Acordo de Paris. 2015b. Disponível em https://www.un.org/en/climatechange/paris-agreement. Acesso em: 06 mai. 2021.

Emissions Gap Report 2020. Nairobi, 2020. Disponível em: https://www.unep.org/pt-br/emissions-gap-report-2020. Acesso em: 06 mai. 2021.

Global Sustainable Development Report 2019: The Future is Now - Science for Achieving Sustainable Development. New York, 2019a. Disponível em: https://sustainabledevelopment.un.org/content/documents/24797GSDR report 2019.pdf. Acesso em: 06 mai. 2021.

The Sustainable Development Goals Report. 2019b. Disponível em: https://unstats.un.org/sdgs/report/2019/The-Sustainable-Development-Goals-Report2019.pdf. Acesso em: 06 mai. 2021.

Transformando Nosso Mundo: A Agenda 2030 para o Desenvolvimento Sustentável. 2015a. Disponível em: http://nacoesunidas.org/pos2015/agenda2030/. Acesso em: 06 mai. 2021.

SHAYANI, Rafael Amaral. O papel crucial dos professores para conter a emergência climática. UnB Agência, Brasília, 13 dez. 2019. Disponível em: https://noticias.unb.br/artigos-main/3777-o-papel-crucial-dos-professores-para-conter-aemergencia-climatica. Acesso em: 06 mai. 2021.

Efeito da pandemia na educação superior: pedra de tropeço ou degrau para o progresso? UnB Agência, Brasília, 11 mai. 2020a. Disponível em: https://noticias.unb.br/artigos-main/4125-efeito-da-pandemia-na-educacao-superior-pedrade-tropeco-ou-degrau-para-o-progresso. Acesso em: 06 mai. 2021.

O novo formato das aulas remotas deve motivar os estudantes para objetivos nobres, elevada determinação e intenso esforço. UnB Agência, Brasília, 06 jul. 2020b. Disponível em: https://noticias.unb.br/artigos-main/4266-o-novo-formato-dasaulas-remotas-deve-motivar-os-estudantes-para-objetivos-nobres-elevada-determinacaoe-intenso-esforco.Acesso em: 06 mai. 2021.

Novos Paradigmas para o Planejamento Energético: A Importância das Fontes Renováveis e da Eficiência Energética. In: FRONTIN, Sergio de Oliveira (Org). Ações de Eficiência Energética Associadas à Geração Distribuída Estudo de Caso: Campus Darcy Ribeiro da Universidade de Brasília. 2. ed. Brasília: Editora Casa 73, 2020c. p. 303-316.

A importância de estudar Mudanças Climáticas na graduação.

UnB Agência, Brasília, 12 abr. 2021. Disponível em: https://noticias.unb.br/artigosmain/4886-a-importancia-de-estudar-mudancas-climaticas-nos-cursos-de-graduacao.

Acesso em: 06 mai. 2021.

UNESCO. Engineering: Issues, Challenges and Opportunities for Development. 2010. Disponível em: http://www.unesco.org/new/en/natural-sciences/sciencetechnology/engineering/engineering-education/unesco-engineering-report/. Acesso em: 06 mai. 2021. 
. Engineering Education: Transformation and Innovation. 2014.

\begin{abstract}
Development Devel//unesdoc 2021.
\end{abstract}

Engineering for Sustainable Development: Delivering on the Sustainable

\title{
THE AWAKENING OF STUDENTS SELF-MOTIVATION THROUGH PROJECT-BASED LEARNING CONSIDERING SUSTAINABLE DEVELOPMENT GOALS
}

\begin{abstract}
The adoption of a more student-centered education requires motivation techniques to make the transition from expository class, with the teacher as the source of knowledge, to classes in which the student is the protagonist of his own learning. Empowering students to improve the world based on technical engineering knowledge is an effective way of motivation. The use of project-based learning, in which students have the freedom to choose their own project theme, as long as it is related to the United Nations Sustainable Development Goals, include them in a world-wide effort of noble goals, motivating them to study as they see the importance of engineering disciplines to improve the world. This learning approach was carried out in two undergraduate engineering classes of basic of electricity. As a result, all students reported that this methodology helped them to develop several engineering skills.
\end{abstract}

Keywords: Project-based learning; Student-centered education; Sustainable Development Goals. 